\title{
Method for Estimating Thread Strength Reduction of Damaged Parent Holes with Inserts
}

\author{
David L. Johnson* and Troy C. Stratton ${ }^{\dagger}$ \\ ATK Thiokol Inc., Brigham City, UT 84302
}

\begin{abstract}
During normal assembly and disassembly of bolted-joint components, thread damage and/or deformation may occur. If threads are overloaded, thread damage/deformation can also be anticipated. Typical inspection techniques (e.g. using GO-NO GO gages) may not provide adequate visibility of the extent of thread damage. More detailed inspection techniques have provided actual pitch-diameter profiles of damaged-hardware holes. A method to predict the reduction in thread shear-out capacity of damaged threaded holes has been developed. This method was based on testing and analytical modeling. Test samples were machined to simulate damaged holes in the hardware of interest. Test samples containing pristine parent-holes were also manufactured from the same bar-stock material to provide baseline results for comparison purposes. After the particular parent-hole thread profile was machined into each sample a helical insert was installed into the threaded hole. These samples were tested in a specially designed fixture to determine the maximum load required to shear out the parent threads. It was determined from the pristine-hole samples that, for the specific material tested, each individual thread could resist an average load of 3980 pounds. The shear-out loads of the holes having modified pitch diameters were compared to the ultimate loads of the specimens with pristine holes. An equivalent number of missing helical coil threads was then determined based on the ratio of shear-out loads for each thread configuration. These data were compared with the results from a finite element model (FEM). The model gave insights into the ability of the thread loads to redistribute for both pristine and simulated damage configurations. In this case, it was determined that the overall potential reduction in thread load-carrying capability in the hardware of interest was equal to having up to three fewer threads in the hole that bolt threads could engage. Onehalf of this potential reduction was due to local pitch-diameter variations and the other half was due to overall pitch-diameter enlargement beyond Class 2 fit. This result was important in that the thread shear capacity for this particular hardware design was the limiting structural capability. The details of the method development, including the supporting testing, data reduction and analytical model results comparison will be discussed hereafter.
\end{abstract}

\section{Nomenclature}

A $\quad=$ Average ultimate load per thread $(3,984 \mathrm{lbs} /$ thread...see Equation 1$)$

$\mathrm{B}=$ Percent average ultimate load per thread (6.7\% ultimate load/thread...see Equation 2 )

$\mathrm{C}=$ Equivalent missing threads (see Equation 3 )

\section{Introduction}

A s a matter of good engineering practice parent-hole thread shear capability exceeds the tensile capability of the A threaded fastener. In some cases thread shear capability may be less than the tensile capability of the fastener due to geometric design constraints. When this occurs thread shear strength is typically assessed utilizing an ultimate strength approach with appropriate factor of safety. The primary assumption to this approach is sufficient material ductility such that all threads share the load equally at time of first thread failure (uniformity around the

Copyright 2005, ATK Thiokol Inc., affiliate of Alliant Techsystems Inc., published by CPIA with permission

*Engineer, RSRM Nozzle Design, LE1.

${ }^{\dagger}$ Senior Engineer, RSRM Nozzle Design, LE1. 
thread circumference is also assumed). An additional assumption is that the thread stress state is dominated by shear.

Uniform threads (unified thread form) result in non-uniform load distribution in elastic materials. Typically the top few threads carry the majority of the load. In order for the aforementioned primary assumption to hold the material must be capable of sufficient elasto-plastic behavior to allow for thread load redistribution to uniformthread loads prior to first thread failure (the assumption is invalid for brittle materials). This typically requires on the order ten percent uniaxial ductility for uniform threads. Locally deviated thread diameters may result in the need for greater ductility to fully redistribute the thread loads. In some cases bending stresses in the threads may have an impact on thread shear strength. This paper illustrates a case of insufficient thread load redistribution in a limited ductility material (7075-T7351 aluminum) with locally deviated thread diameters due to assembly/disassembly thread damage. Strength reductions from thread bending stresses are also evident.

During assembly and disassembly of threads with interference-type lock features (locking inserts, lock patches, etc.), thread damage and/or diametral deviation may occur in parent holes. If threads are overloaded and subsequently chased with a tap, further thread deviation can also be anticipated. Typical inspection techniques (e.g. using GO-NO GO gages) may not provide adequate visibility of the extent of thread damage in the lower portions of threaded holes. A more detailed Johnson-Gage inspection technique of individual thread diameters has provided actual pitch-diameter profiles of damaged holes. This more detailed approach provided insights into the overall condition of the threaded holes. Examples of measured pitch diameters of actual damaged threaded holes are shown in Figure 1. Each hole exhibits local diameter deviations with respect to the general thread diameter. These holes were all deemed acceptable from using a "GO-NO GO" gage inspection method.

An approach was developed to estimate the potential reduction in thread shear-out capability of the damaged threaded holes in this material with limited-strain capability. This method was linked to damage actually measured in the hardware through testing and analytical modeling.

\section{Materials}

Shear-out samples were machined from a portion of 2-inch diameter bar stock made of 7075-T7351 aluminum. The samples were designed such that the engagement length of the installed insert was constant. The threads in the test samples were CNC mill single-point machined to control the magnitude and location of the thread deviations in the samples. A variety of thread pitch-diameter profiles were tested in an attempt to approximate some of the conditions found in hardware parent holes. These configurations (referred to as series) are summarized in Table 1. Examples of the specified CNC thread-profiles for Series E-H are shown pictorially in Figure 2. These configurations have the smaller deviations, on the order of 0.003 inches radially. Series J-M have the same profiles but the deviations are on the order of 0.005 inches radially. The samples were tested as shown in Figure 3 . Figure 4 shows a piece of bar stock, a pre-test sample and a post-test sample. Figure 5 shows the parent-hole pitch-diameter measurements of the Series F samples. Due to machining tolerances the center-bulge region was typically machined with a +0.002 -inch offset-from-target value for both small and large deviation series.

\section{Experimental Procedures}

This investigation involved testing and computer simulations. These two methods were combined to derive an equivalent thread-damage model.

\section{A. Shear-Out Testing}

The thread shear-out capability was determined experimentally for a variety of samples. These samples were manufactured with various thread profile configurations. The thread-profile configurations were selected in an attempt to approximate some of the thread damage measured in hardware components. A test fixture was designed specifically for this test effort. The fixture simulated failing the threads by pulling through a hole of a specified diameter. Actual hardware bolts and helical inserts were used in the test setup. These samples were loaded into the test fixture and tested to failure as shown in Figure 3.

\section{B. Equivalent Thread Damage}

The following equations were used for determining the number of equivalent missing threads from the experimental shear-out data:

$$
A=\frac{\text { Average Load of Series } A \text { (Pristine) }}{\text { Number of Threads Engaged }}=\frac{59,760 \mathrm{lbs}}{15 \text { threads }}=3,984 \mathrm{lbs} / \mathrm{thread}
$$




$$
\begin{aligned}
& B=\left(\frac{A}{\text { Average Load of Series } A(\operatorname{Pr} \text { istine })}\right) * 100=\left(\frac{3,984 \mathrm{lbs} / \text { thread }}{59,760 \mathrm{lbs}}\right) * 100=6.7 \% / \text { thread.(2) } \\
& C=\left(1-\frac{\text { Series Average Load (e.g. B, C...Q) }}{\text { Average Load of Series A(Pr istine) }}\right) / B
\end{aligned}
$$

Equation 1 was used to calculate the baseline average ultimate load per thread using the second Series A (Pristine-Constrained) data. Note: There are two Series A. The first series tested had samples with unconstrained helical insert ends. There were two problems with these samples: (1) the test-fixture bolt tended to bind up in the helical insert during fixture assembly, and (2) the upper insert thread would spring out (disengage) causing lower ultimate loads. The sample configuration was altered to include a 0.10 -inch counter bore at the insert major diameter both above and below the helical insert to contain any intentionally disengaged coils. All of the second "Series A" samples had higher shear-out values than the first Series A samples, which can be seen in the figures discussed in "Results" section below. It should be noted that a baseline of 15 threads ( 0.83 inches) of engagement was used.

Equation 2 is a further refinement of Equation 1. Equation 2 was used to calculate the baseline average percentage of the ultimate shear load that each thread resists. This value is used in Equation 3. Equation 3 was used to calculate the reduction of load-carrying capability for each of the thread-profile series in terms of equivalent number of "missing" threads.

Table 1 - Shear-out Series Configuration Summary

\begin{tabular}{||c|l||}
\hline \hline Series & \multicolumn{1}{|c||}{ Description } \\
\hline A & Reference/undamaged \\
\hline B-1 & Undamaged - target 1 bottom thread removed \\
\hline B-2 & Undamaged - target 2 bottom threads removed \\
\hline B-3 & Undamaged - target 3 bottom threads removed \\
\hline C & Undamaged "oversized" hole (with tap) with standard insert \\
\hline D & Bottom 2-3 threads oversized with tap \\
\hline E & Small top taper (single point milled thread profile) \\
\hline F & Small top taper \& center bulge (single point milled thread profile) \\
\hline G & Small top \& bottom taper (single point milled thread profile) \\
\hline H & Small top \& 2/3 down bulge (single point milled thread profile) \\
\hline J & Large top taper (single point milled thread profile) \\
\hline K & Large top taper \& center bulge (single point milled thread profile) \\
\hline L & Large top \& bottom taper (single point milled thread profile) \\
\hline M & Large top \& 2/3 down bulge (single point milled thread profile) \\
\hline N & Undamaged with 2 insert coils (removed prior to installation) \\
\hline P & Oversized Parent Hole with Oversized Helical Inserts \\
\hline Q & Twinsert Helical Inserts \\
\hline
\end{tabular}




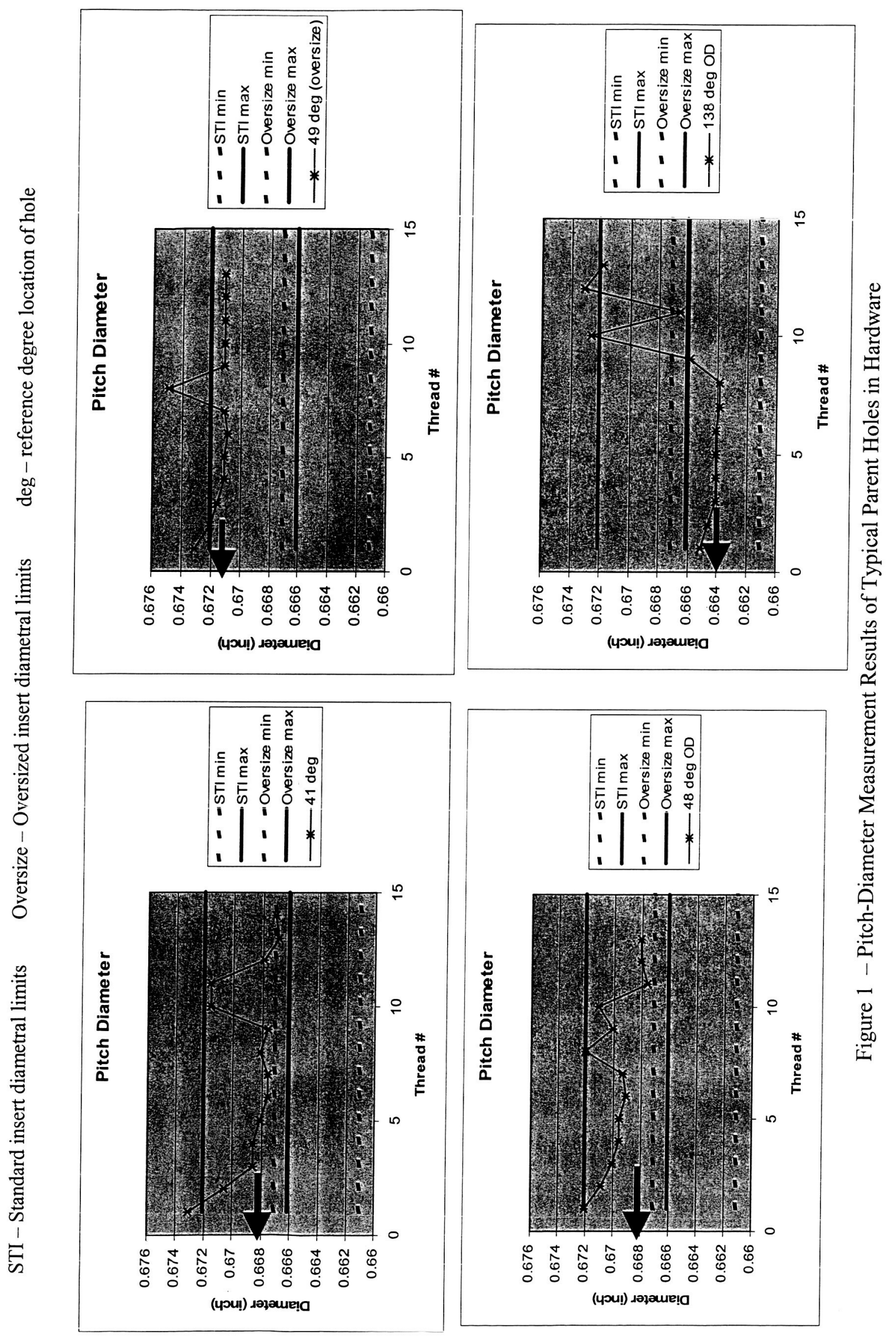

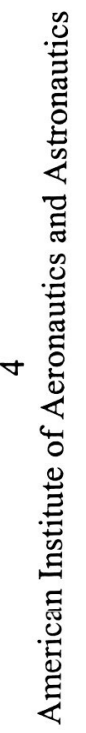


Tapered Hole at Top Only

$(0.006 \mathrm{D})$

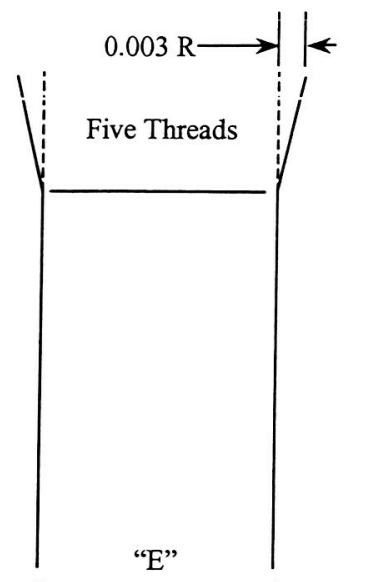

Tapered Hole at Top $(0.006$

D) \& in Middle (0.006 D)

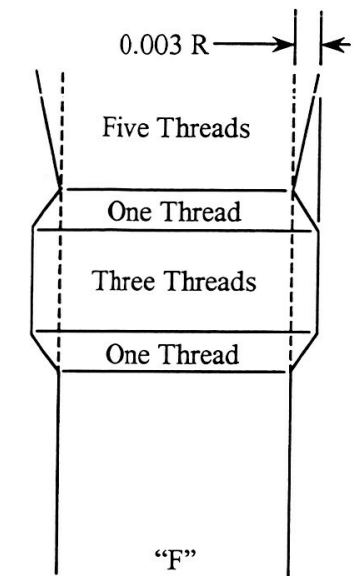

Tapered Hole at Top $(0.006$

D) \& at Bottom (0.006 D)

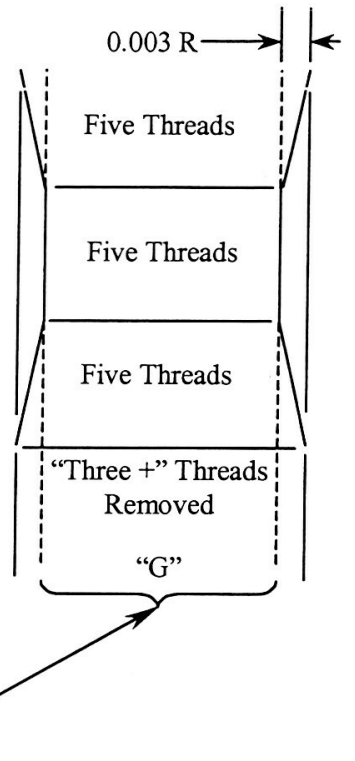

.

Nominal Pitch Diameter 


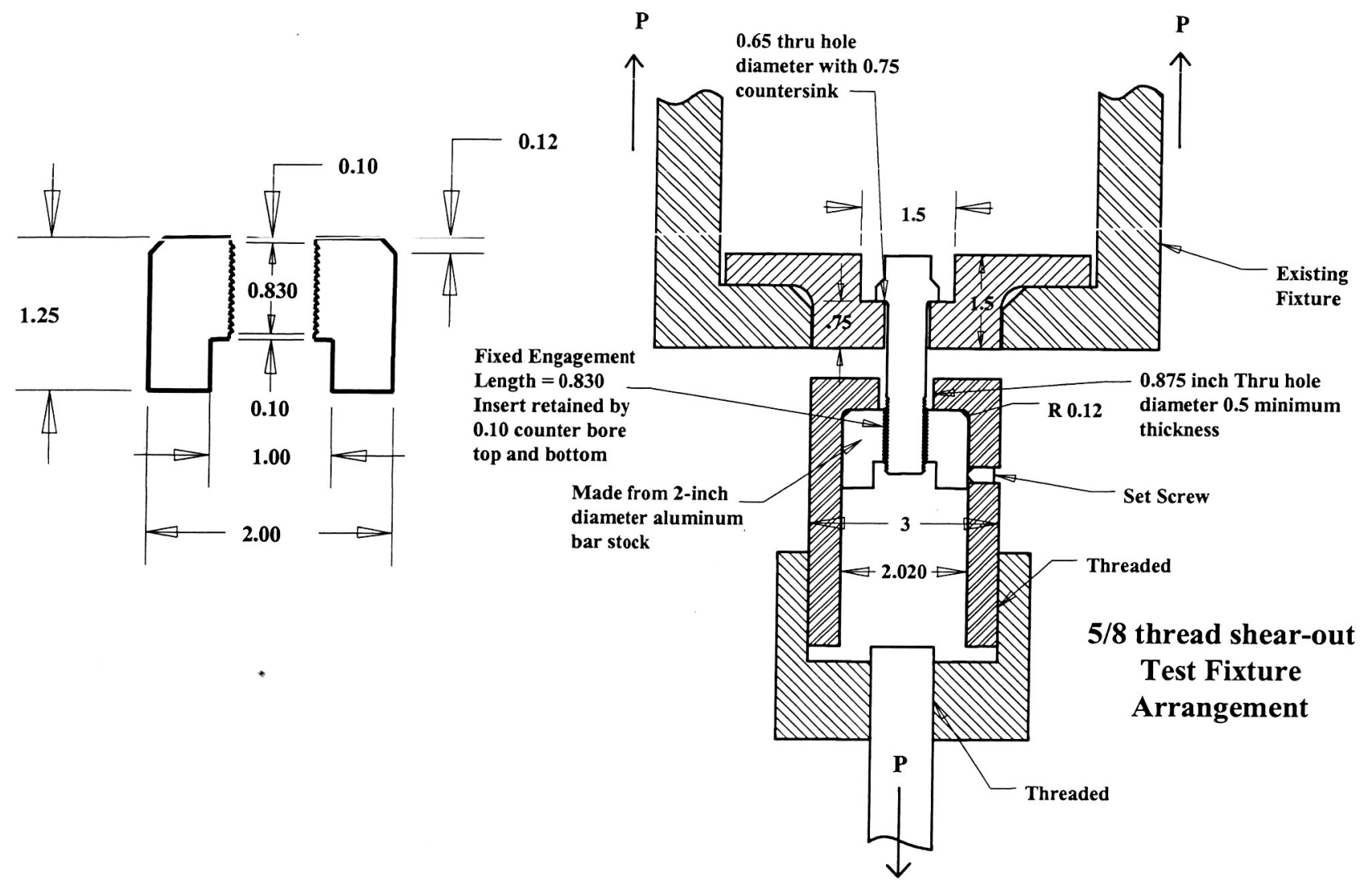

Figure 3 - Thread Shear-out Testing Configuration

\section{Thread FEA}

Some thread configurations were also simulated in a Finite Element Model (FEM). The FEM effort involved several steps. First, a geometrically parametric axisymmetric surface-to-surface contact model was developed to represent a through-hole with a helical insert installed and a corresponding bolt inside the insert as shown in Figure 6.

The parametric model eased the analysis of various pitch-diameter profile configurations. The primary purpose of the finite element analysis (FEA) was to study thread load distributions and magnitude of plastic straining in individual threads. Based on FEA results, test observations and some judgment a thread fit or "tightness" weighting function was created. The function was employed as part of categorizing the degree and propensity of occurrence of damage in actual threaded hardware parent holes using actual pitch diameter measurements.

The model was elasto-plastic with an assumed stress-strain curve for aluminum shown in Figure 7. Elastic behavior was assumed for steels (bolt and insert). Surface-to-surface contact was employed for the thread surfaces. A baseline run was made for a "pristine" thread condition to a pre-calculated failure load of $45,700 \mathrm{lbs}$. The equivalent plastic strain versus thread depth is shown at failure load in Figure 8. The top threads at the left side of the graph were at 15 to $25 \%$ strain. Using this strain level as a failure indicator other configurations were analyzed to determine estimated failure loads. Figure 9 shows resulting individual thread loads and estimated failure loads (see figure legend) for some example thread deviation configurations. 
The reduced failure loads were equated to missing threads and in conjunction with individual thread loads, were used as part of the input to finalize the subsequent weight function shown in Figure 10.

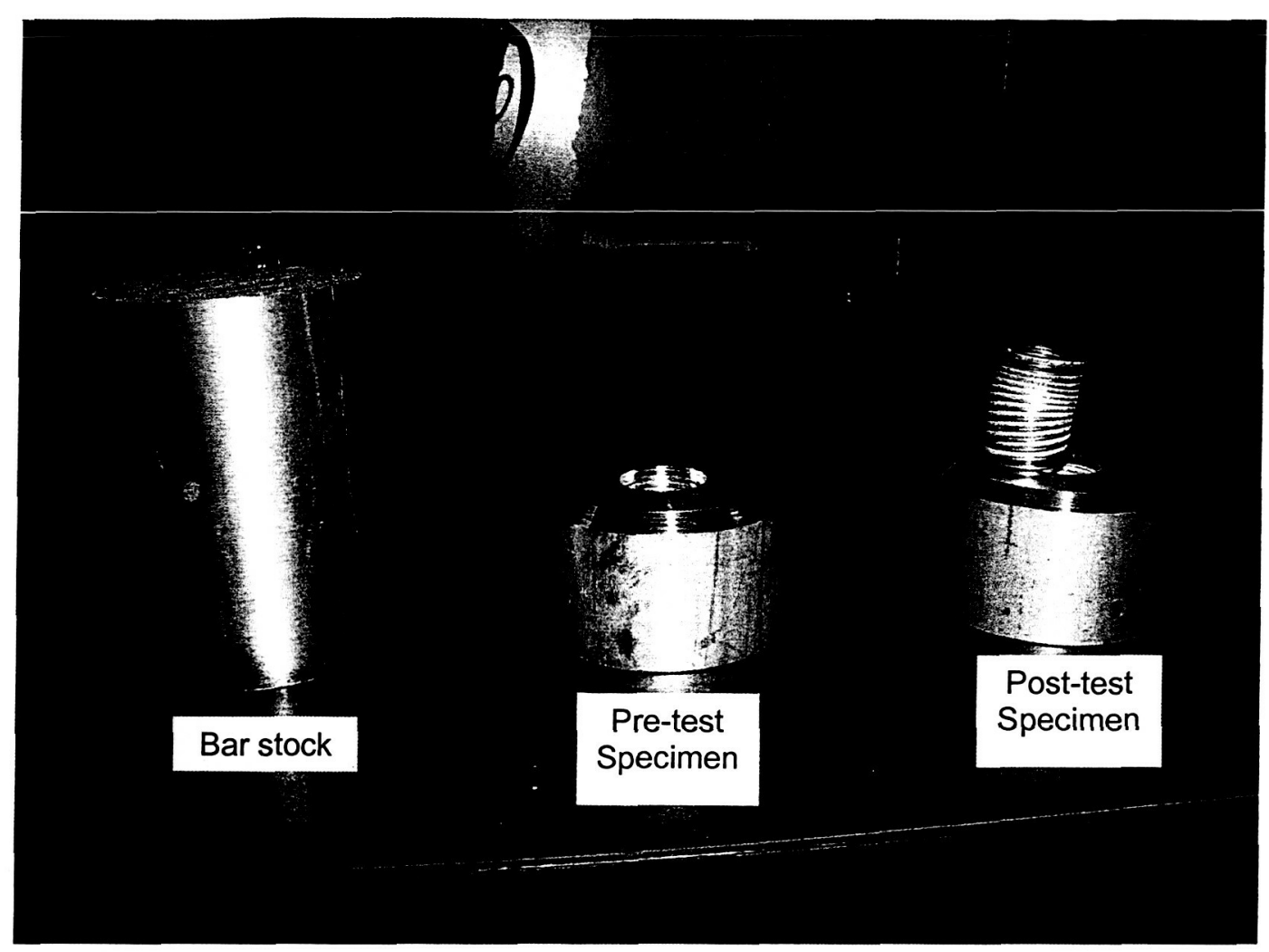

Figure 4 - Examples of Bar Stock and Shear-Out Specimens

\section{Results}

\section{A. Shear-Out Testing}

The ultimate shear-out test results are summarized in Table 2. The shear-out loads of the holes having deviated pitch diameters were compared to the ultimate loads of the specimens with pristine (uniform) threads. An equivalent number of missing helical coil threads was then determined based on the ratio of shear-out loads for each thread configuration to the uniform condition (restrained A series). The equivalent number of missing helical coil threads results are summarized in Table 3. This comparison is shown graphically in Figure 11. Figure 12 shows a graphical summary of the equivalent missing thread values in increasing order. It can be seen that the samples having holes machined with small pitch-diameter variations had minimal load reduction and no significant equivalent missing threads $(0.0-0.5)$. All configurations having large pitch-diameter variations, particularly toward the bottom of the hole, exhibited significant capability reduction. As anticipated the configurations with actual missing bottom threads (either helical insert or parent hole) had the most significant and predictable load reductions.

\section{B. Thread Analysis}

The degree of thread damage varied from hole to hole. A "general" measure of the magnitude of damage and the propensity of occurrence was desired for input to subsequent full scale testing with simulated thread damage. Because the top threads inherently take the most load, the "tightness" of a thread fit is generally more important the deeper the thread is in the hole. This is particularly true for limited ductility materials in order for the lower threads to adequately share load prior to first thread failure. In an effort to account for this, the first step to assessing the criticality of a damaged or enlarged thread was, as previously discussed, to assign an "importance" or tightness weight factor to each thread. The relative tightness was measured as the deviation of an individual thread with respect to the "typical" pitch diameter for that hole determined by judgment of what the hole diameter would have 
been without damage. Figure 1 shows some examples of measured pitch diameter with arrows indicating the "typical" diameter of each hole. Note how individual thread diameters deviated from the typical pitch values.

The upper threads inherently load faster than the lower threads and can be relatively "looser" than lower threads and have equal impact on strength. A solution was to assign a "weight" to threads as shown in Figure 10 . The initial assumed weight function distribution was based on roughly inverting a typical elastic thread load distribution. The final distribution (equation) reflected judgment based on input from the elasto-plastic FEM with threads explicitly modeled and from the test results. This was accomplished by assuming that individual or local thread damage is proportional to the "weighted" area under the deviation distribution. An example deviation distribution for a hole is shown in Figure 13. Fach individual thread area is multiplied by the weight factor from Figure 10 and then summed. The resulting summation was then multiplied by a proportionality factor to convert the summed damage value to a "score" or ranking value. The proportionality factor was arbitrarily chosen so that scores varying between zero and 2000 represented zero to two missing threads. By applying this approach to the FEA analyzed and actual tested profiles and iteratively modifying the weighting equation and score categories until roughly the same number of equivalent missing threads were indicated, the scores or rankings in Table 4 and weight function in Figure 10 were established. The method was then employed to rank or score the actual hardware holes based on data from 183 sample measured available holes.

\section{Equivalent Thread Damage}

From measured pitch diameters in the 183 holes, the data were assessed to estimate the distribution of typical pitch diameters and using established scoring method estimate the local thread damage.

Figure 15 shows the distribution of the typical pitch diameters. The results indicated that:

(1) $85 \%$ of holes were "enlarged" from wear and tear beyond initial drawing requirements (Class 3 ). The wear and tear is a result of using locking inserts, lock patch with inserts and general thread chasing with taps.

(2) $60 \%$ of holes were beyond Class 2 fit allowances with a standard (STI) insert.

(3) Some instances of greater than Class 2 diameters allowances in conjunction with oversize inserts \& Twinsert (helical insert within a larger helical insert).

Results 2 and 3 demonstrate an inherent outcome due to the screening limitations of GO/NO-GO inspections. The inspections are not always sufficiently accurate to prevent all out-of-tolerance conditions from occurring. These results have an impact on the thread capacity.

A capability reduction of up to 1.5 threads for an STI insert installed in "oversized" hole (based on Series C shown in Figure 16) from general diameter enlargement was observed. The reduction was attributed to the increasingly significant thread separation (moment arm) between the bolt and parent hole pitch diameters resulting in insert "thread rolling". The thread rolling results in local parent thread bending stress in addition to the shear stress.

Series P (oversized hole with oversized insert) indicated a similar reduction again attributed to insert thread rolling. Series Q (Twinsert configuration) also indicated some reduction even though thread shear calculations indicate a slight increase in capability from shearing at a larger diameter including the effect of the inner insert engaging one thread less than the outer insert. The reduction is again attributed to thread bending induced from outer insert thread rolling.

Since $60 \%$ of the holes fell into categories similar to Series C, P and Q assuming strength reduction of up to 1.5 equivalent threads was appropriate to compensate for typical pitch diameter growth.

The estimated distribution of the local damage magnitude is shown in Figure 14. From the results it was estimated that:

(1) $52 \%$ of the holes had less than $1 / 2$ equivalent threads of damage

(2) $87 \%$ of the holes had less than 1 equivalent thread of damage

(3) $13 \%$ of the holes had more than 1 equivalent thread of damage

(4) $3 \%$ of the holes had more than $1 \frac{1}{2}$ equivalent threads of damage.

(5) There was no indication of a significant probability of 2 or more equivalent threads of damage in any hole.

The local "damage" capability reduction of up to 1.5 equivalent threads was additive to the observed capability reduction due to general pitch diameter enlargements. The net result was an estimated potential varying reduced thread capability up to three equivalent missing threads or $20 \%$ strength reduction in a single hole out of an eighthole bolt pattern. Follow-on reduced thread capacity full-scale testing was then conducted by clipping/removing prescribed numbers of coils in each hole baised on the estimated propensity and magnitude of strength reductions established by this method. 


\section{Conclusion}

An estimate of the propensity and magnitude of reduced capability was completed. It was determined that the overall estimated potential reduction in thread load-carrying capability in the material of interest was varying reduced capability of up to three fewer threads in a single hole of an eight-hole pattern. Half of the potential reduction was from unscreened general thread enlargement and half from local thread damage. The testing also demonstrated that clipping/removing coils from the insert is a repeatable simple method to induce a reduced thread capacity equivalent to removing threads. The testing showed that the ability of threads to carry the applied load (i.e. capacity) can be influenced by several factors such as:

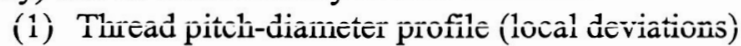

(2) Material shear strain capability (ability of highest strained thread to "hold out" until lowest loaded thread(s) reaches full load

(3) Local insert thread "rolling" which can be exacerbated by loose fitting threads (typical pitch diameter beyond Class 2 fit).

Reducing thread capacity based on estimates of propensity and magnitude of thread capability reduction was used to simulate bounding damage scenarios in follow-on full-scale test hardware.

\section{Acknowledgments}

The Authors would like to thank NASA-MSFC (Marshall Space Flight Center) for their continued support and interest in our desire to further understand issues related to the hardware's reusability and performance.

\section{References}

${ }^{1}$ Advertisement, "Thread Keeps Missions from Falling Apart" Aerospace Engineering, Volume 24, Number 10, SAE International, November 2004, p. 8, (graphical representation of thread loading).
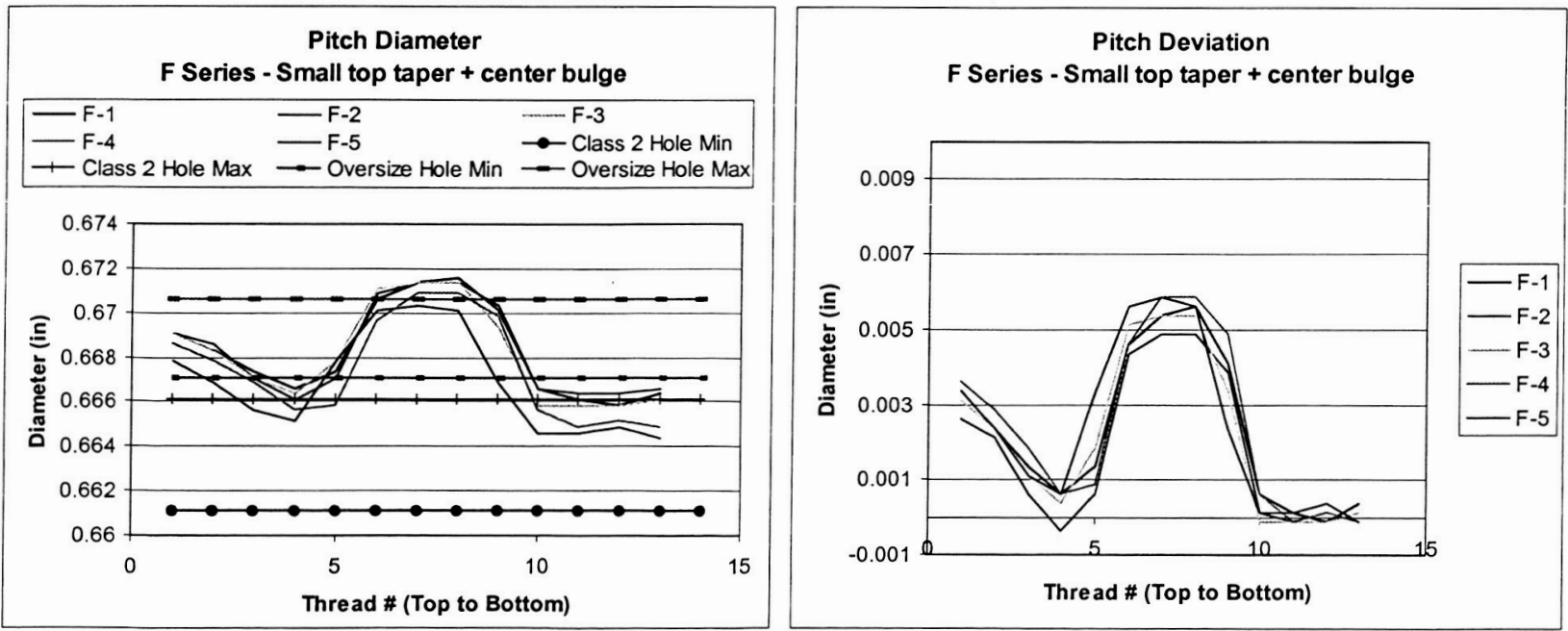

Figure 5 - Pitch Diameter Variation of Series F Test Samples 


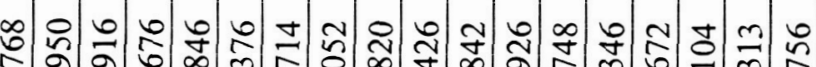
nो

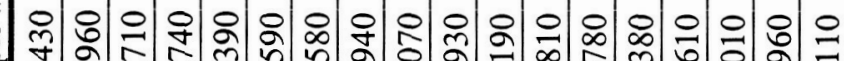

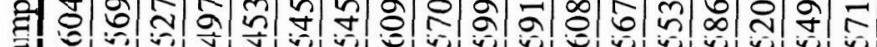

क

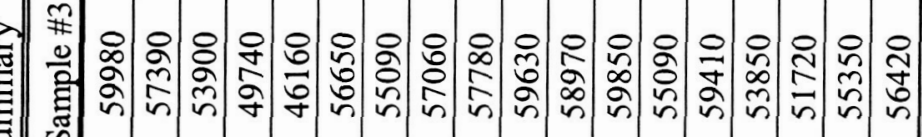

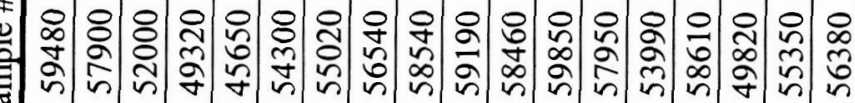

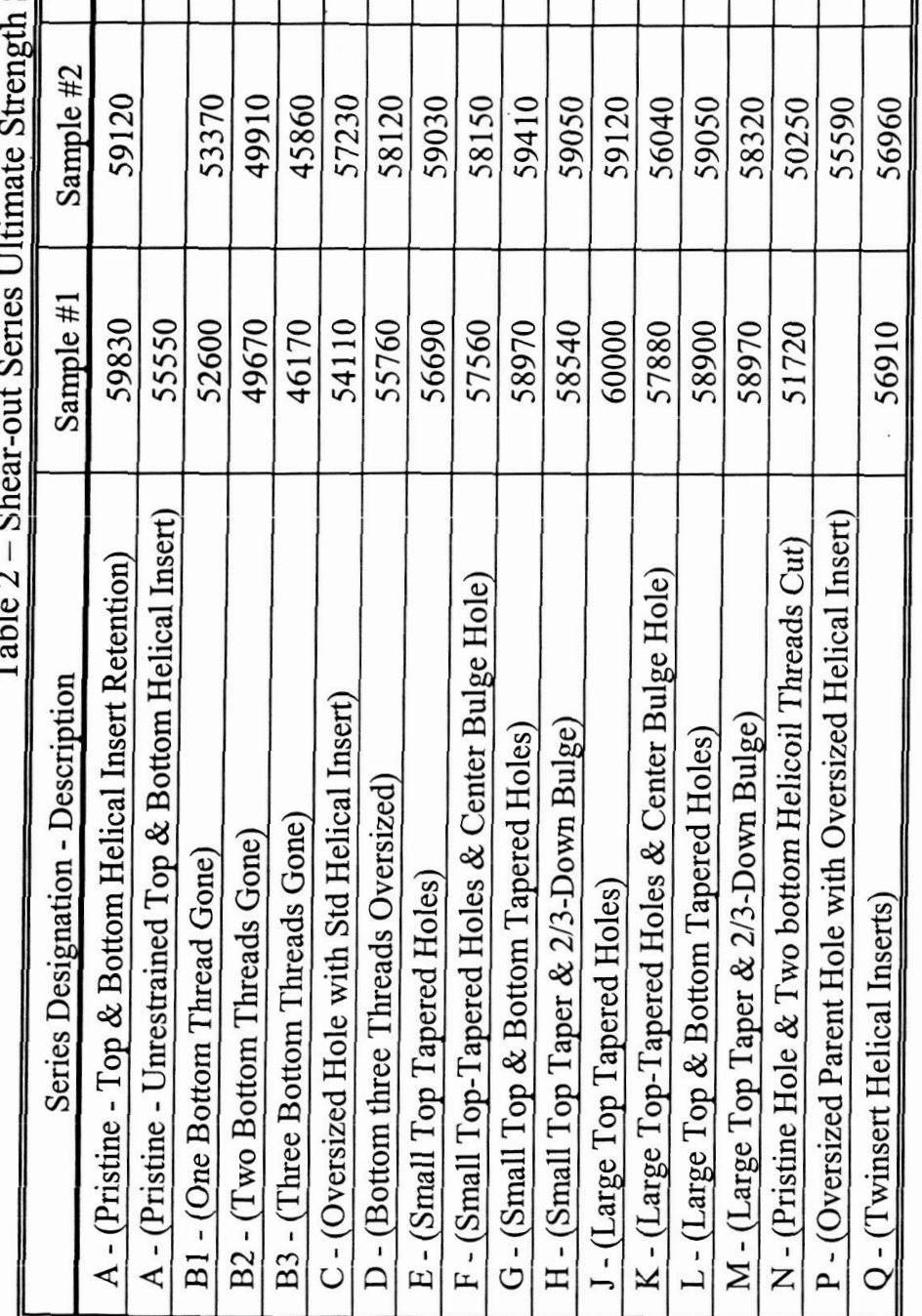



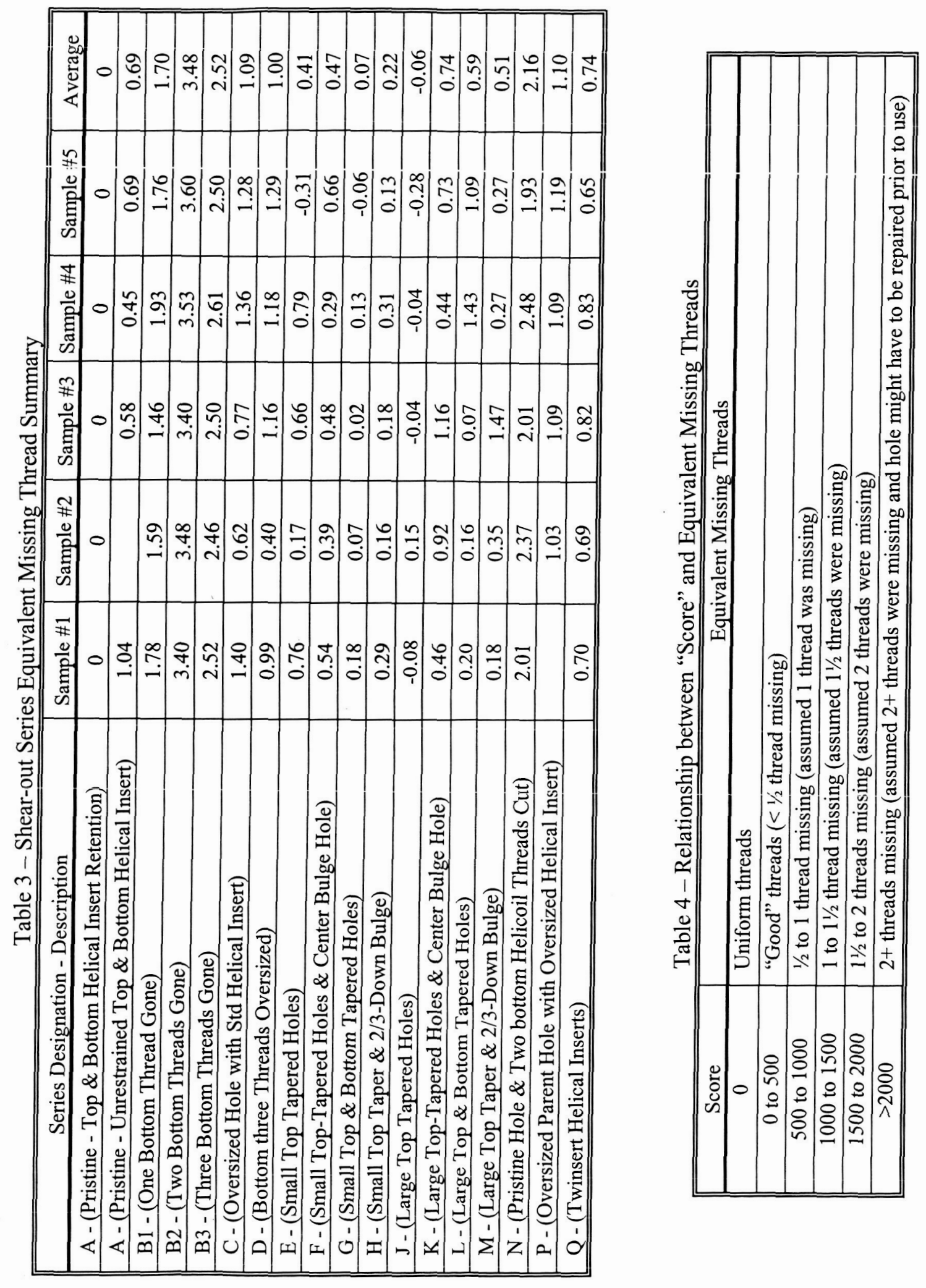

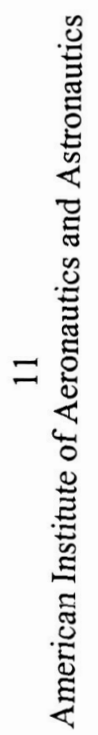




$$
\bar{z}
$$




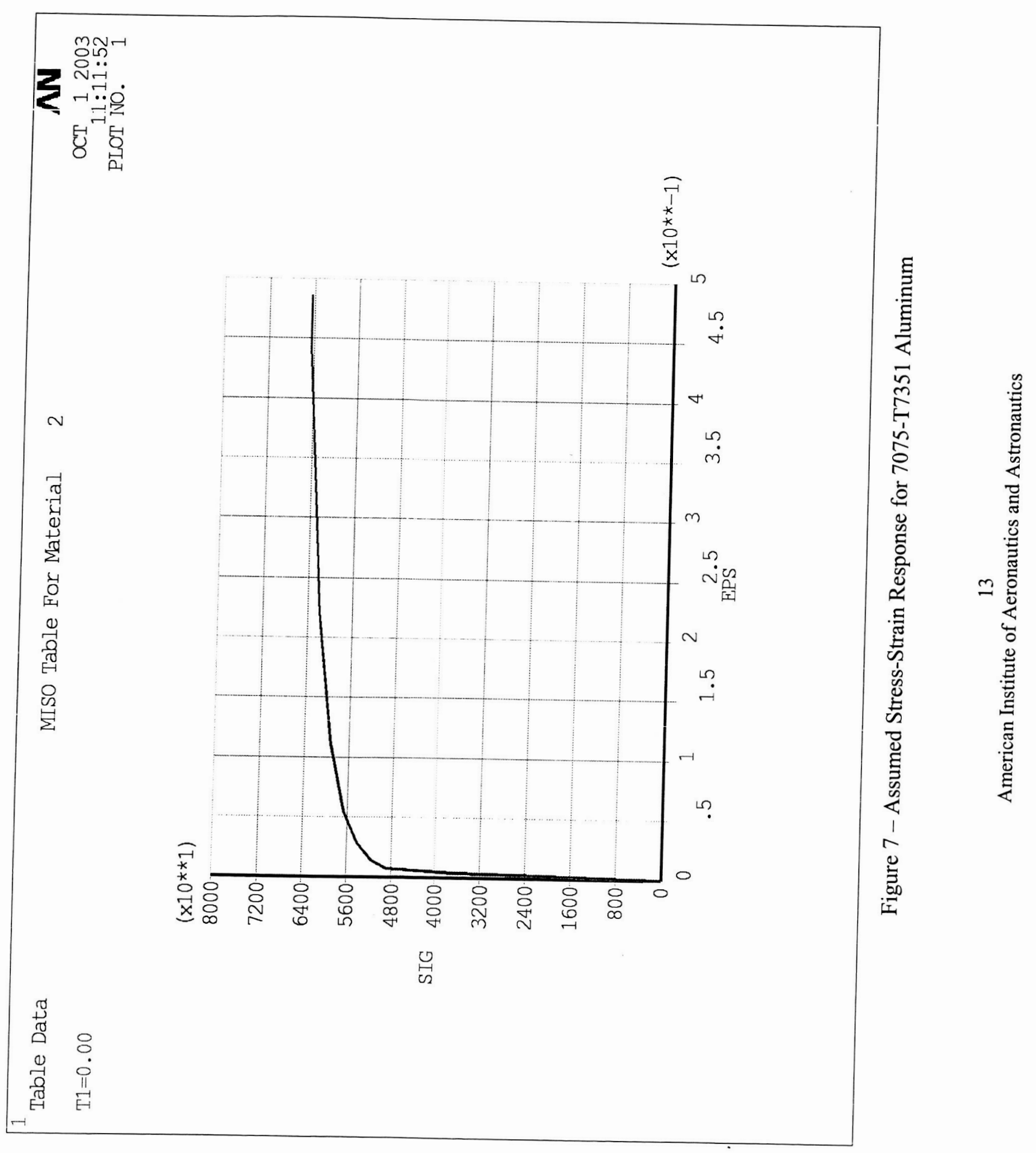




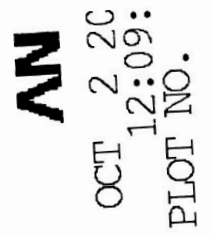

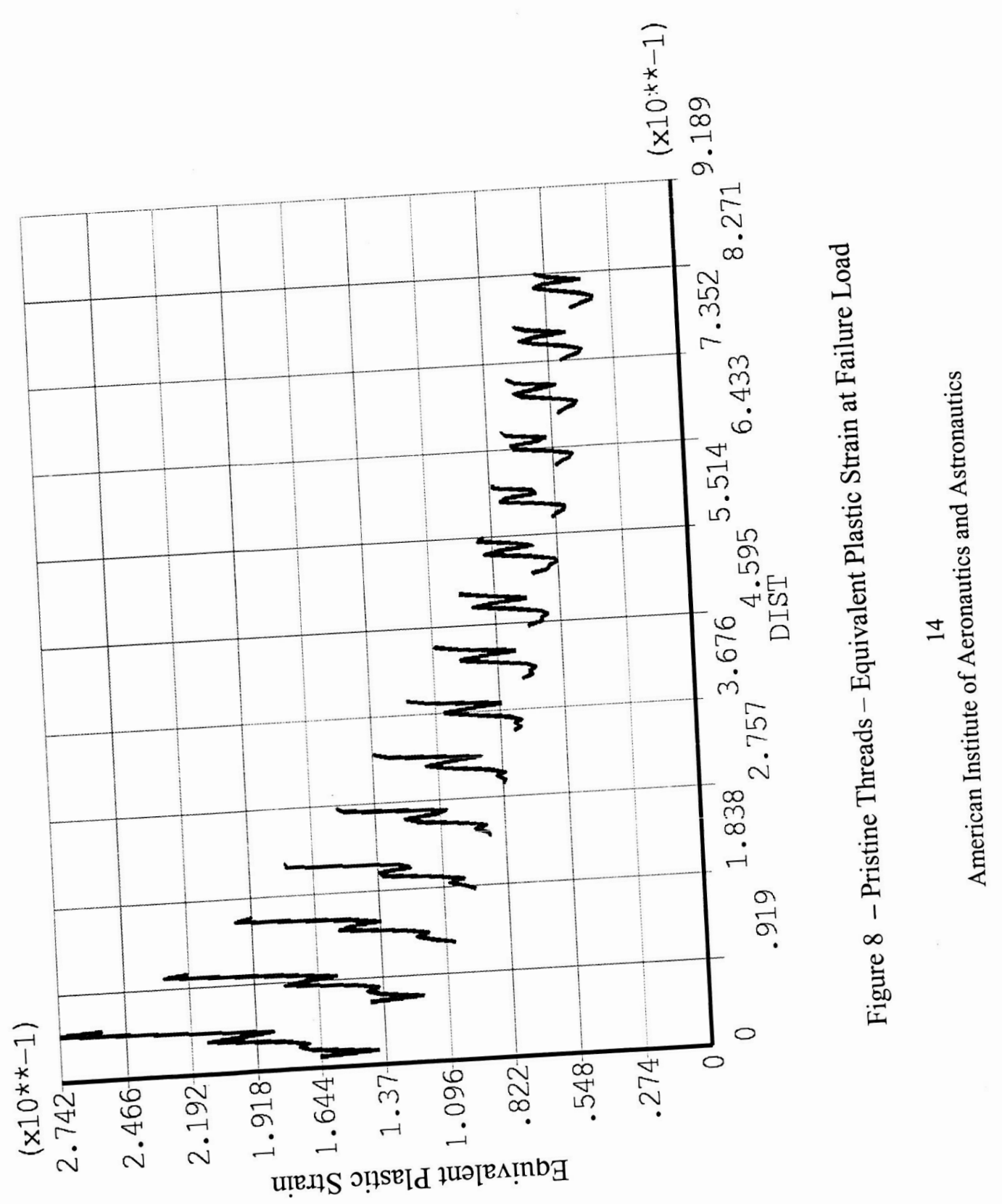

덩

놈

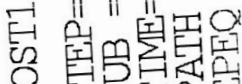

O 


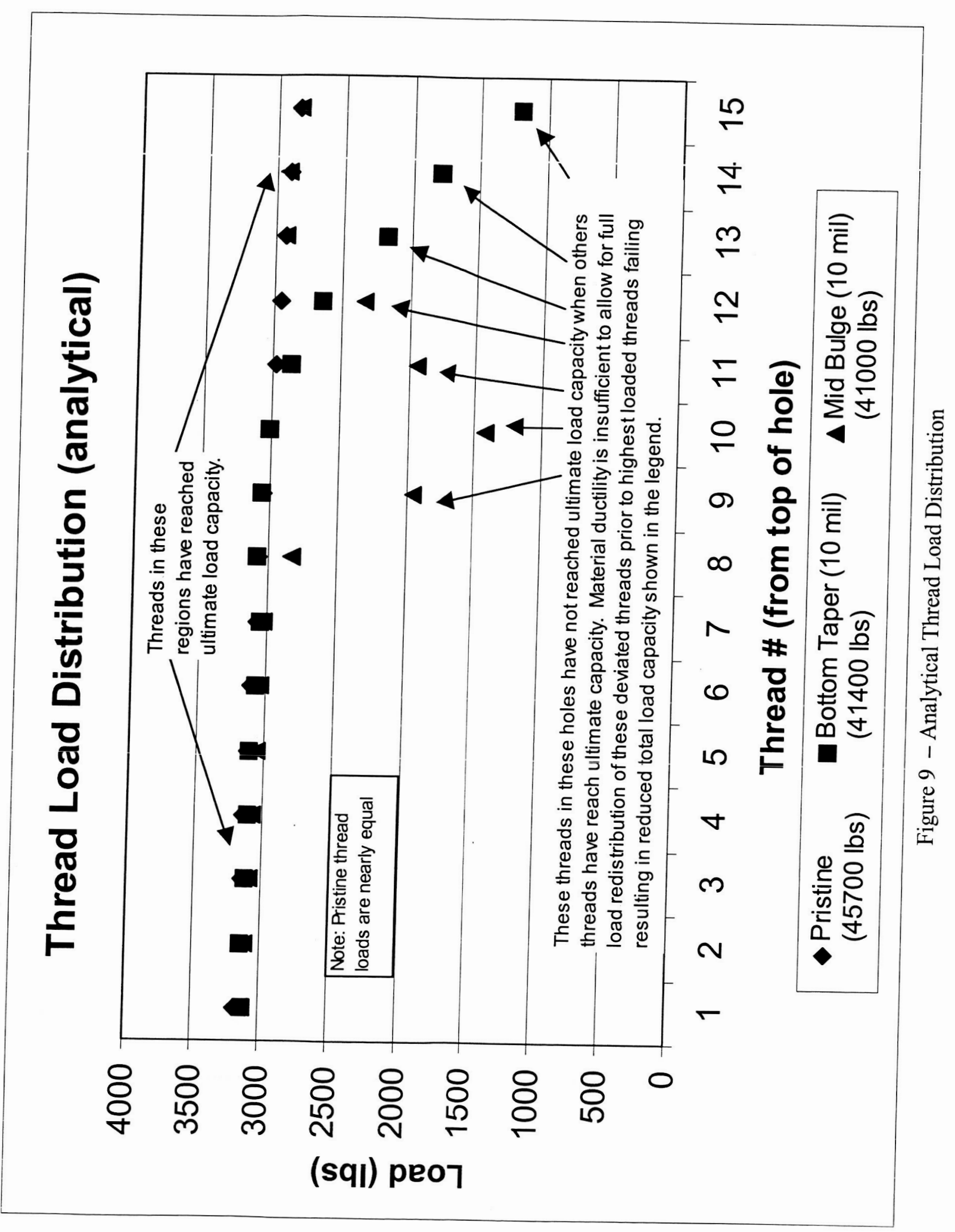




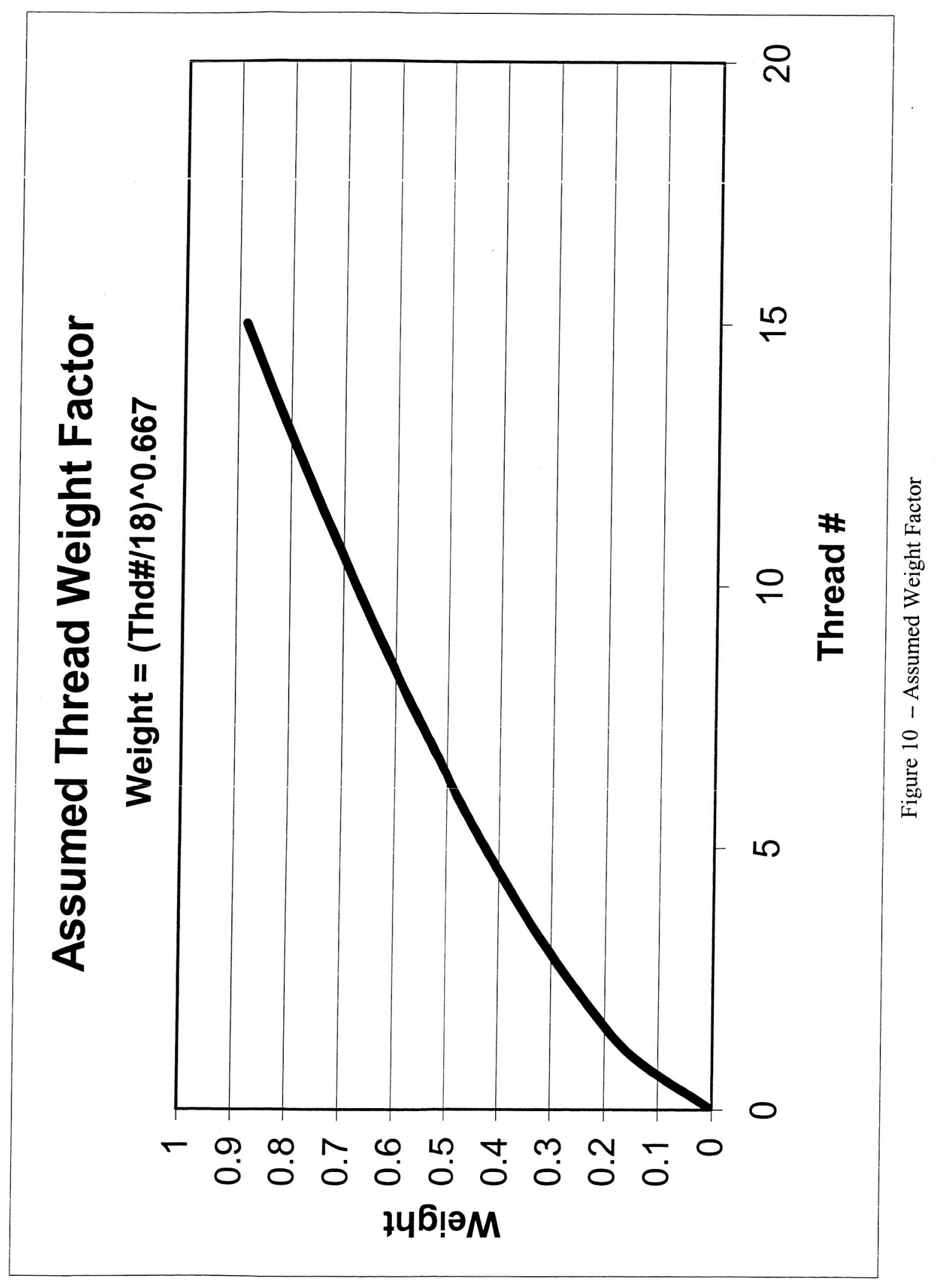




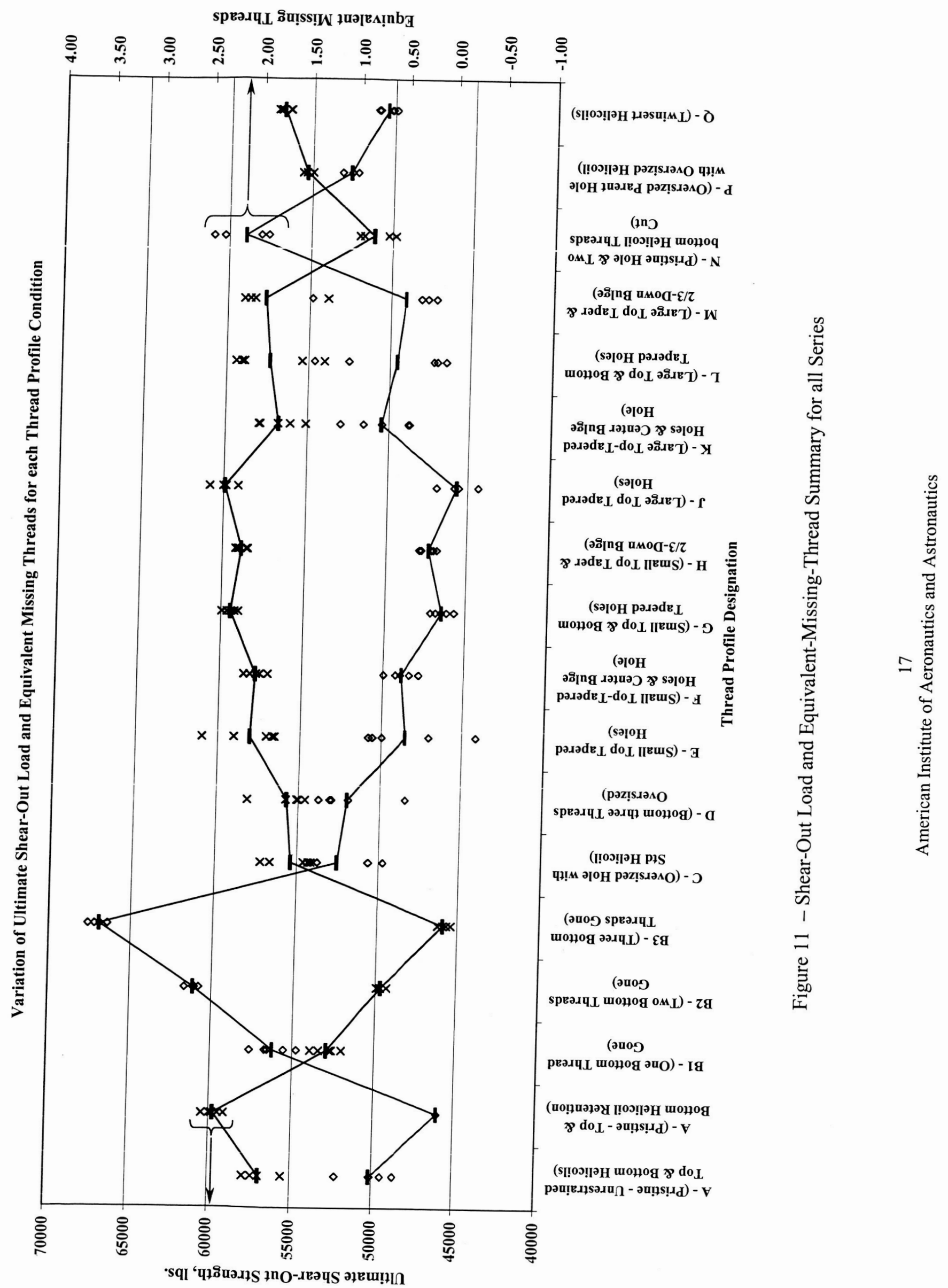




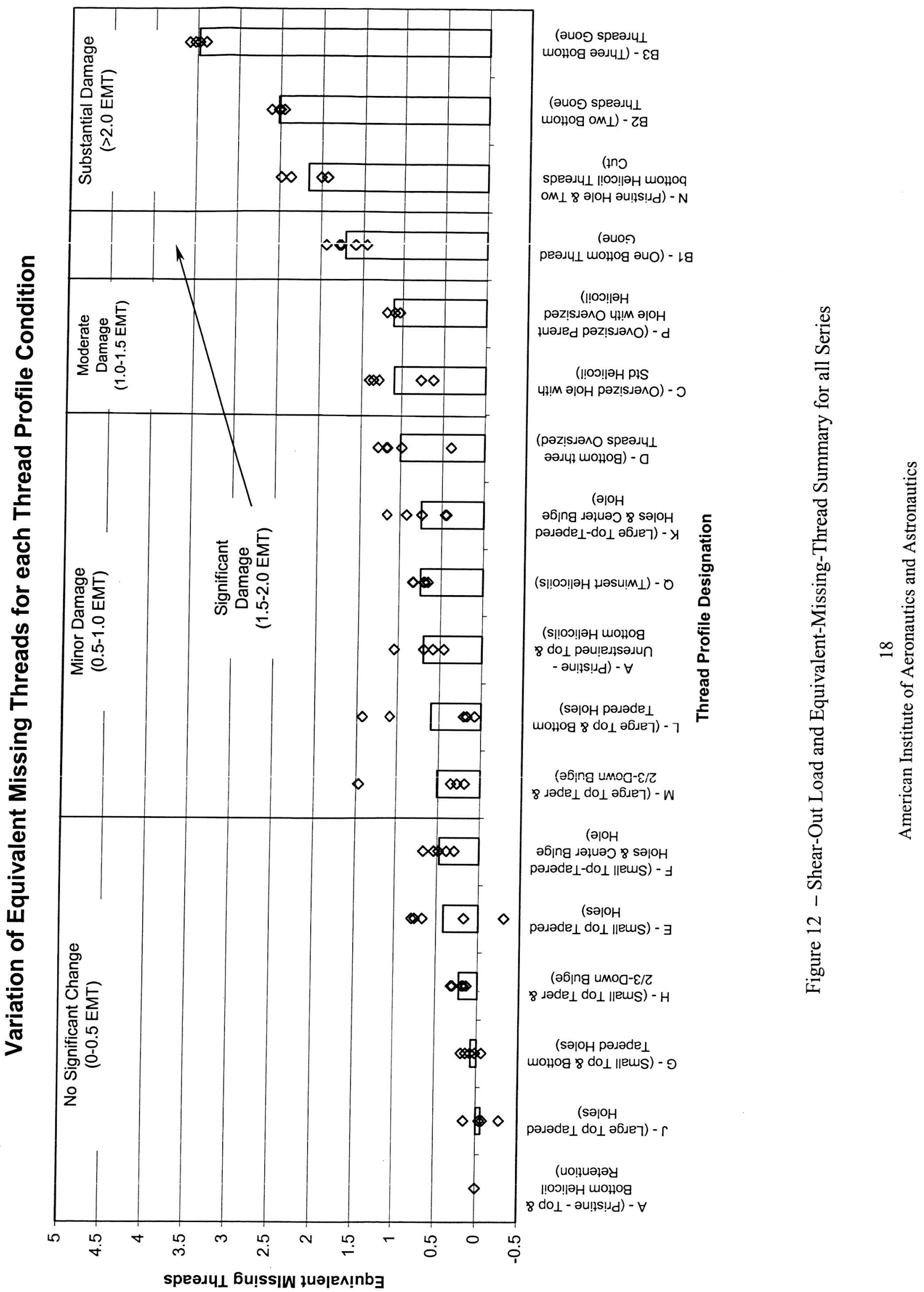

